This paper was published in IEEE Transactions on Industrial Electronics in Jan. 2015, doi 10.1109/TIE.2014.2327581, is available at:

http://ieexplore.ieee.org/stamp/stamp.jsp?tp=\&arnumber=6823665

C. Gan; R. Todd, J.M. Apsley, "Drive System Dynamics Compensator for a Mechanical System Emulator," IEEE Transactions on Industrial Electronics, vol. 62, no. 1, pp. 70-78, Jan. 2015, doi: 10.1109/TIE.2014.2327581

(C) 2014 IEEE. Personal use of this material is permitted. Permission from IEEE must be obtained for all other uses, in any current or future media, including reprinting/republishing this material for advertising or promotional purposes, creating new collective works, for resale or redistribution to servers or lists, or reuse of any copyrighted component of this work in other works. 


\title{
Drive System Dynamics Compensator for a Mechanical System Emulator
}

\author{
C. Gan, R. Todd, Senior Member, IEEE and J. Apsley, Member, IEEE
}

\begin{abstract}
A system compensator is designed to cancel the mechanical characteristics of a power electronics-controlled induction motor drive with a coupled electrical generator system. The emulation scheme using the compensator enables accurate emulation of high speed and high power mechanical system dynamics. The compensator is developed based on the system transient response of the test rig, considering the full operating range of the test system. The design process of the compensator is described and it is validated in both the time and frequency domains. Finally, the effectiveness of the compensator is demonstrated by simulation and experimental emulation of aero gas engine dynamics.
\end{abstract}

Index Terms-Hardware-in-the-loop, aero gas engine emulation, dynamics compensator, system identification

\section{INTRODUCTION}

$\mathrm{T}$ HE use of hardware-in-the-loop (HIL) techniques based on an electrical drive system is common in mechanical system emulation. It can allow complex dynamic systems to be accurately emulated in a safe, repeatable and cost effective way. A commercial electrical drive system acts as the emulator and a real-time platform (RTP) contains a mathematical model of the system to be emulated [1]. The electrical drive may be employed to emulate either a mechanical power source or load [2]-[7]. Emulating mechanical systems with electrical drives using HIL techniques enables the experimental demonstrators to be used to gain vital insight into electro-mechanical interactions in systems which consist of multiple power sources, generation systems and loads.

Mechanical power source emulation is widely used in transportation and renewable applications such as combustion engines [8], aero gas turbines [2], [7], [9], and wind turbines [4], [5], [10], [11]. Recent dynamic emulation research uses a model-based control method, in which a speed/torque controller is designed for the emulator system, taking into account the emulated mechanical model. The control bandwidth of the emulator system should be high enough to allow good emulation of the dynamics of interest. An auto-tuned PI speed controller was used in [8] to allow the induction motor to emulate an internal combustion engine (ICE) system. In [10], [11], a hysteresis current control and a PID speed controller were designed and implemented to obtain a wider control bandwidth in comparison with that of a conventional PI control, to enable the emulation of the dynamics of a wind turbine drivetrain. The emulation of conventional mechanical systems using an electrical drive system is relatively straightforward because the power electronics-controlled machines are dynamically fast. However, the designed controller is limited to a specific system and cannot accommodate applications with faster dynamics.

In [2], a PI speed controller was designed to allow the shaft dynamics of a twin-shaft gas engine to be emulated with a synchronous motor. When the emulated engine inertia decreases, the emulation performance is reduced. [9] presented the accuracy and stability issues for the emulation of a faster aero engine using the speed controller which is designed for a slow engine case. All studies suggest that the model-based emulation method is limited to a specific dynamic system and a redesign of the controller is required for each new application.

Previous electrical drive based mechanical load emulation research was mainly based on either an inverse mechanical dynamics approach [12] or a feedforward speed-tracking control scheme [3], [13], [14]. The inverse load dynamics method [12] provides acceptable open-loop emulation; however, it cannot be used in the closed-loop control system due to the violation of the pole-zero structure of the emulated mechanical load [3]. The feedforward speed-tracking control method uses a compensator, based on the analytical drive system transfer function, to eliminate the drive control loop dynamics. This approach can preserve the model mechanical dynamics and give very good closed-loop emulation results; however, the compensator's effectiveness is only verified through the emulation performance of fairly slow and low power dynamics at modest speeds. The bandwidth of the emulated linear loads (less than $0.2 \mathrm{rad} / \mathrm{s}$ ) in [3] is well within that of the uncompensated drive system while the emulated nonlinear mechanical applications in [13], [14] also exhibit relatively slow dynamics (around 25rad/s). In Section III, the analytical compensator from [3] is analyzed and discussed, showing that the analytical estimated drive control characteristic cannot accurately represent the real system dynamics, especially during the high frequency and high speed range.

In this paper, an electrical drive based mechanical system emulation approach is proposed, which uses an advanced compensator design using test data to extend the emulation capability of a physical hardware system without the need for any hardware changes or knowledge of any drive system parameters. The compensator is designed by applying parametric system identification [15] to experimental data from an induction machine drive step response, to cancel the natural drive speed control loop dynamics. The compensator was 
enhanced to incorporate the field weakening effects of the machine drive and demonstrated by emulating an aero gas engine. The method proposed in this paper is not limited to a specific mechanical system model and is particularly suitable for the emulation of the high speed and high power mechanical systems such as an aero gas engine.

\section{EXPERIMENTAL TEST FACILITY}

A subset of the aircraft power system facility [7] is shown in Fig. 1 with a focus on the gas engine high pressure (HP) spool emulation hardware. The dynamic behaviour of the spool is emulated using a dSPACE real-time platform (RTP) containing the gas engine model, and an $115 \mathrm{~kW}$ commercial vector controlled induction machine (IM) drive. In the existing set up of the RTP the mechanical system model is represented by a thermodynamic and mechanical aero gas engine, which receives the flight profile data, such as environmental data and throttle (pilot's lever angle (PLA)), measured electrical generating torque on both spools, $T_{G e n}$ and measured drive speed, $\omega_{r}$ as inputs. The engine model in the RTP then outputs HP and LP spool speed references to the relevant electrical drive unit. The low pressure (LP) spool emulator is not shown but it has a similar configuration.

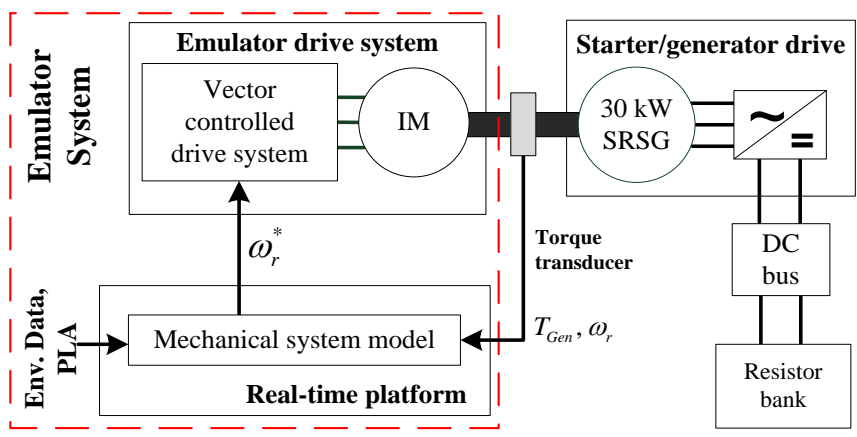

Fig. 1. Block diagram of the HP spool emulation test facility

Candidate electrical generators for embedding in an aircraft gas engine are coupled to the relevant spool emulator and the dynamic and steady-state load profiles are implemented using active load systems and resistive load banks which are connected to the generators through a reconfigurable DC network. A 30kW, 15,000rpm switched reluctance starter generator (SRSG) is connected to the HP spool emulator drive system.

\section{Proposed Emulation Technigue}

In this section, the emulation schemes using the model-based control design and analytical compensator design are analysed and discussed. An advanced emulation approach, applying system identification techniques to experimental data, is then described and the corresponding compensator design procedure is presented.

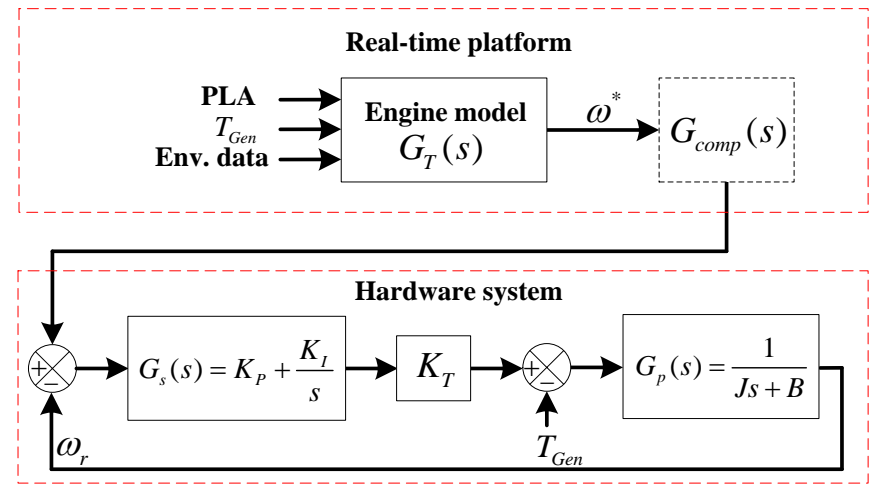

Fig. 2. Block diagram of the compensator implementation

\section{A. Emulation using Analytical Identified Compensator}

When detailed information for an emulator system, including machine parameters, control structure and tuning values, is available, an inverse analytical transfer function based compensator can be designed as explained in [3], [13]. The compensator is designed to cancel the natural emulator system dynamics so that the physical causality of the emulated system is preserved.

Fig. 2 illustrates the structure of the emulation system where the feedforward compensator $G_{\text {comp }}$ is implemented in the RTP between the emulated aero gas engine model $G_{T}$ and the emulator hardware system. The IM drive dynamics in Fig. 2 are dominated by the mechanical characteristics of the speed loop. Since the current loop in the drive system is operating much faster than the outer speed control loop, the current loop and power converter dynamics can be neglected. $G_{s}$ refers to the PI speed controller of the drive system with $K_{T}$ representing the machine torque constant. The transfer function $G_{P}$ describes the rotor dynamics of the test rig.

Assuming $G_{\text {comp }}=1$ and $T_{G e n}=0$ in Fig. 2, the relation between the engine model output speed dynamics $\omega^{*}$ and the drive system output $\omega_{r}$ can be derived as:

$$
\frac{\omega_{r}}{\omega^{*}}=G_{T F}=\frac{G_{s} K_{T} G_{P}}{1+G_{s} K_{T} G_{P}}
$$

In order to obtain a good emulation, the condition in (2) must be fulfilled over the bandwidth of the emulated aero gas engine model, $G_{T}$.

$$
\frac{\omega_{r}}{\omega^{*}} \approx 1 \text {, when } G_{s} K_{T} G_{p}>>1
$$

This requires the speed regulator to be designed taking into account the dynamics of both $G_{P}$ and $G_{T}$.

When the term $G_{\text {comp }}$ is in place, the system input-output relation becomes:

$$
\frac{\omega_{r}}{\omega^{*}}=G_{\text {comp }} \frac{G_{s} K_{T} G_{P}}{1+G_{s} K_{T} G_{P}}=1
$$



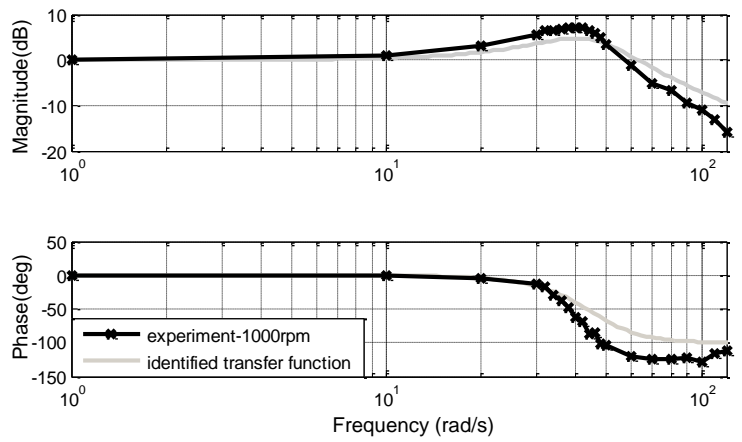

Fig. 3. Experimental and analytical estimated frequency response comparison

where

$$
G_{\text {comp }}=G_{T F}^{-1}=\frac{1+G_{s} K_{T} G_{P}}{G_{s} K_{T} G_{P}}
$$

An analytical description, (5), of $G_{T F}$ in (4) can be obtained with the estimated parameters of the drive system. However, in practice, the accuracy of the parameter estimation has been found to affect the performance of the analytical compensator. To derive the analytical system transfer function as defined by (5) (assuming a PI structure), control tuning values $\left(K_{P}, K_{I}\right)$ are required together with the inertia, $J$, friction coefficient, $B$ and IM drive torque constant, $K_{T}$.

$$
G_{T F}=\frac{K_{P} K_{T} s+K_{I} K_{T}}{J s^{2}+B s+K_{p} K_{T} s+K_{I} K_{T}}
$$

It has been found that estimation errors in the rig inertia, $J$ and torque constant, $K_{T}$ have a big effect on the accuracy of $G_{T F}$. In the HP spool emulation facility, the system inertia was found from experimental tests to an accuracy of $\pm 10 \%$. The torque constant, $K_{T}$ is calculated in the drive using a fixed motor efficiency of $90 \%$, which may lead to an estimation error when the motor efficiency deviates significantly from $90 \%$. The other HP spool drive parameters are given in the Appendix. Fig. 3 shows the frequency response measured in the experimental system at 1000rpm together with the response of (5) based on the estimated parameters in the Appendix. The experimental results were obtained by superimposing a 10rpm sinusoidal component on the 1000rpm base value. The frequency of the sine wave was varied from 1 to $120 \mathrm{rad} / \mathrm{s}$.

Fig. 3 shows a mismatch between the measured and estimated frequency response above 10rad/s. The estimated resonant peak is $3 \mathrm{~dB}$ lower in magnitude and $4 \mathrm{rad} / \mathrm{s}$ higher in frequency than the measured resonant peak of $8 \mathrm{~dB}$ at $40 \mathrm{rad} / \mathrm{s}$. After reaching the resonant frequency, the roll-off rate also differs with a higher gradient in the measured response.

Below 10rad/s, the system is capable of reasonable system emulation without the need of a compensator. Above 10rad/s, a compensator must be used, however, the analytical model is not accurate enough for the compensator design due to the unmodelled system dynamics and the variation in $K_{T}$, due to the field weakening, and magnetic and current saturation effects, which makes the model less accurate for the high speed and high power region. The analytical compensator is suitable only for slow dynamics emulation below machine base speed and so, this method is not suitable for the emulation of the high speed and high power applications such as an aero engine.

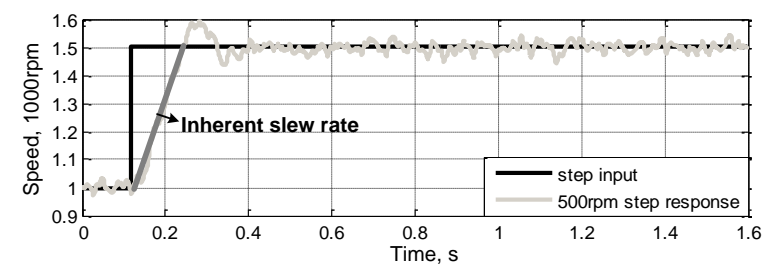

Fig. 4. HP spool drive system step response from 1000 to $1500 \mathrm{rpm}$ at no-load

\section{B. Proposed Emulation Compensator}

In order to derive a better compensator, which can capture the unmodelled drive system dynamics, the parametric system identification approach is applied to simple experimental system step responses. System identification is generally applied to experimental results using statistics theory [15]. It constructs a mathematical model of a dynamic system, which can reproduce the test system input-output properties. For parameter system identification, either frequency or time domain transient response data can be used [15]. Due to the fact that the frequency response method involves time consuming experiments and requires an expensive network analyser [16], the step response based transient analysis is preferred. It was performed using the System Identification Toolbox in Matlab [17].

\section{1) Transient response selection}

Fig. 4 shows the drive speed response to a 500rpm step, from $1000 \mathrm{rpm}$ to $1500 \mathrm{rpm}$. The 500rpm step test was chosen because it can give a proper persistent excitation to the drive system within the bandwidth of interest. It should be noted that the step input is internally limited to a slew rate of $3759.4 \mathrm{rpm} / \mathrm{s}$ by the drive system.

\section{2) Model structure selection}

The structure of the identified transfer function model depends on how the relationship between the input, output and noise is described. The Output-Error (OE) model structure is defined by (6), where $y(k)$ is the system output, $u(k)$ is the system input, $e(k)$ is the white noise and $B(q) / F(q)$ is the transfer function to be estimated.

$$
y(k)=\frac{B(q)}{F(q)} u(k)+e(k)
$$

The Output-Error model was selected because it allows $B(q) / F(q)$ to be parameterised independent of the noise model. This kind of model structure can also offer an easier identification process and good performance [18].

3) Signal Pre-filtering

From Fig. 4, some low frequency noise exists in the motor step response, which should not be reproduced by the identified transfer function model. The Savitzky-Golay (S-G) filter is a digital smoothing polynomial filter, which was selected as a prefilter because it can preserve the main transient dynamics of the response such as the peak, minima and width [19]. For this study, the polynomial order is 5 and the frame size of the filter is 255 , which gives an excellent preservation of the overshoot dynamics and significantly reduces the low frequency noise effect (beyond 10Hz). 


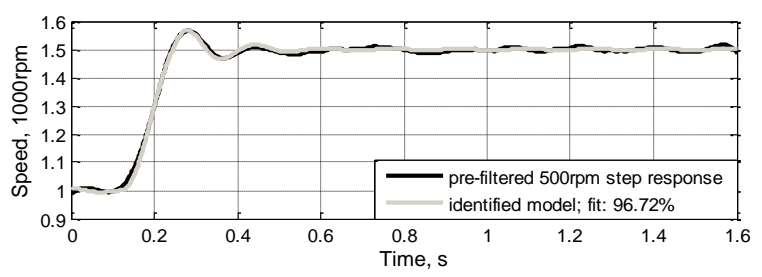

(a) time domain response
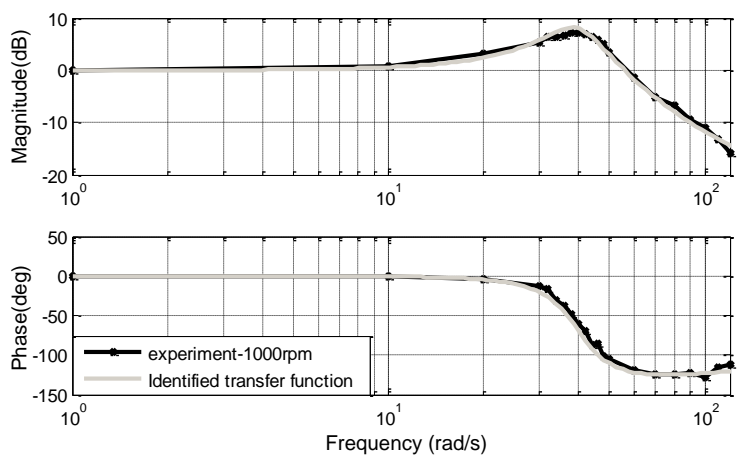

(b) frequency domain response

Fig. 5. Fitting performance of the parametric identified model

\section{4) Model order selection}

The order of both $B(q)$ and $F(q)$ for the OE structure must be specified beforehand. Since the compensator will be implemented in the RTP, computational efficiency is important so a low order model is preferred. After iteratively testing a certain order and checking the fitting performance, a second order, $F(q)$ and first order, $B(q)$ have been selected, which provides a very good model fit, as shown in Fig. 5(a), and matches the orders of the analytical system inner structure, (5).

The fit between the identified transfer function, which in the continuous domain is given by (7), and the measured response is $96.7 \%$.

$$
G_{T F}=\frac{16 s+1569}{s^{2}+17 s+1569}
$$

\section{Influence of Operating Conditions}

Fig. 5(b) shows the frequency response comparison between the identified model and experimental measured data which is the same as that in Fig. 3. From Fig. 5(b), it can be seen that the frequency response from the system identification is much better than that from the analytical model, Fig. 3. However, the compensator, which is the inverse of $G_{T F},(7)$, is improper. An extra term $k s^{2}$, which is related to a certain phase delay, can be introduced into the numerator of (7) to make the inverse of (7) proper and the new $G_{T F}$ becomes:

$$
G_{T F}=\frac{k s^{2}+16 s+1569}{s^{2}+17 s+1569}
$$

The impact of $k$ on (8) should be carefully examined to ensure the system does not become a non-minimum phase system as would happen with (8) when $k<0.008$.

When $k$ is chosen to be small ( 0.01 in this paper), it has been found that the term $0.01 \mathrm{~s}^{2}$ only introduces a small amount of phase delay (maximum $7^{\circ}$ at $120 \mathrm{rad} / \mathrm{s}$ ) to the system over the bandwidth of interest, which has little effect on the emulation performance and can be neglected.
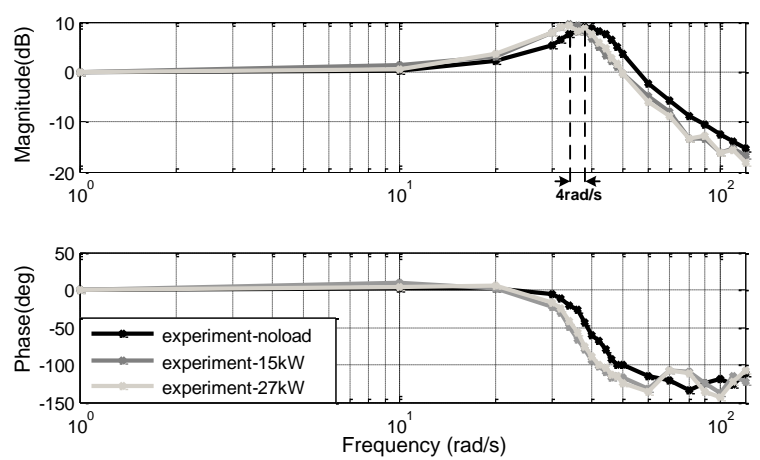

Fig. 6. Experimental frequency response at 9000rpm with variable load
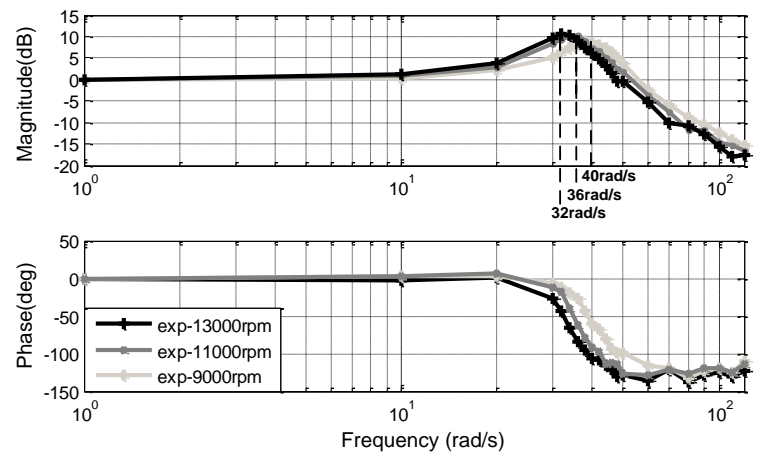

Fig. 7. Experimental frequency response at no load with variable speed

In order to evaluate the drive dynamics dependence on the machine load and speed operating points, a series of frequency chirp tests have been taken under different load and speed conditions. The SRSG generating range is limited to between 8700rpm and 15000rpm [20].

Fig. 6 shows $27 \mathrm{~kW}, 15 \mathrm{~kW}$ and no load uncompensated system responses at $9000 \mathrm{rpm}$ for the current speed controller settings listed in Appendix Table I. From Fig. 6, it can be seen that the resonant frequency in the on-load cases is $4 \mathrm{rad} / \mathrm{s}$ lower than the no-load peak at around 40rad/s with a slightly higher damping. 9000rpm is the lowest speed tested for the generator to ensure the minimum SRSG speed is not exceeded during large load steps. For higher speeds, the generator load has little effect on the system dynamics. Therefore, dynamic dependence on the load is quite minor and so neglected.

Fig. 7 illustrates the dynamic dependence on the machine speed by showing the responses at three different speeds, 9000rpm, 11000rpm and 13000rpm at no load condition. As shown in Fig. 7, the resonant frequency decreases and the system damping increases as the speed increases. In order to include the speed dependence in the identified model, the polytopic structure, which comprises a set of local "small-signal" linear time invariant (LTI) models [21], is sometimes used. However, defining the weighting of separate LTI models is complex and cannot be done analytically. In addition, as the compensator is obtained by inverting the identified system model the polytopic model is not appropriate for this study.

Instead, the theoretical transfer function model of the drive system (1) has been analysed. Due to the field weakening effect, the torque constant, $K_{T}$ will reduce when the machine is 
operating above base speed. The torque constant is inversely proportional to the machine speed, [22], so:

$$
K_{T}^{\prime}=\frac{\omega_{\text {base }}}{\omega_{r}} K_{T}=\alpha K_{T}
$$

where $\omega_{r}$ is the machine speed, $K_{T}^{\prime}$ is the varying torque constant in the field weakening region, $K_{T}$ is the constant value below the machine base speed, $\omega_{\text {base }}$, and $\alpha$ is the ratio of $\omega_{\text {base }} / \omega_{r}$. The effect of field weakening can be considered by combining the $\alpha$ scaling with (5) to give (10), which is valid over the full speed range.

$$
G_{T F}=\frac{\left(K_{P} s+K_{I}\right) K_{T} \alpha}{J s^{2}+B s+\left(K_{P} s+K_{I}\right) K_{T} \alpha}
$$

where

$$
\alpha=1 \text { when } \omega_{\mathrm{r}} \leq \omega_{\text {base }}
$$

$$
\alpha=\frac{\omega_{\text {base }}}{\omega_{\mathrm{r}}} \text { when } \omega_{\mathrm{r}}>\omega_{\text {base }}
$$

Since $B$ is usually at least an order of magnitude less than $K_{P}$, it can be neglected. Then, the $\alpha$ scaling to represent the effect of field weakening can also be used with the system identified transfer function (8) to give (13), which enables the full model response to account for the dependence on the specific operating speed.

$$
G_{T F}=\frac{0.01 s^{2}+(16 s+1569) \alpha}{s^{2}+(17 s+1569) \alpha}
$$

The numerator of (13) is second order and so can be compared to the classic second order differential equation to determine expressions for $\omega_{n}$, and $\xi$. The corner frequency, $\omega_{n}$, of the HP drive system, (14) and the damping factor, $\xi$ both decrease with the machine speed over the base speed, $\omega_{\text {base }}$, which is quite consistent with Fig. 7.

$$
\omega_{n}=\sqrt{1569 \cdot \frac{\omega_{\text {base }}}{\omega_{r}}}
$$

The base speed of the drive is determined by the maximum possible output voltage (dc link voltage) of the inverter and the rated motor parameters [23]. In wide speed range, high power closed-loop controlled induction machine drives the rated speed of the machine is significantly lower than base speed, which is the speed at which the voltage limit of the converter is reached and so field weakening must occur, to enable the load demands to be fulfilled. For the HP spool emulator in Fig. 1 the DC link voltage is $697 \mathrm{~V}$ and the nameplate motor voltage value is $400 \mathrm{~V}_{\mathrm{LLrms}}$. The machine rated line-to-line rms voltage at rated load and slip is calculated to be $330 \mathrm{~V}$ to $385 \mathrm{~V}$ depending on whether the cold or hot value of phase resistance $\left(R_{\text {hot }}=1.4 R_{\text {cold }}\right)$ is considered. For a $55 \mathrm{~A}$ magnetising current and a rated speed of 6745rpm the base speed is estimated to be $8300 \mathrm{rpm}$ (at $400 \mathrm{~V}_{\mathrm{LL}}$ ), $8634 \mathrm{rpm}\left(\right.$ at $385 \mathrm{~V}_{\mathrm{LL}}$ ) and $10073 \mathrm{rpm}$ (at $330 \mathrm{~V}_{\mathrm{LL}}$ ). The variation in base speed due to the effect of temperature on the phase resistance demonstrates the difficulty in accurately analytically determining base speed. The estimated base speed range can be validated using the test data in Fig. 7. The corner frequencies for the three speeds shown in Fig. 7 are plotted in Fig. 8 together with (14), for base speeds of 8300rpm and 10073rpm. The test data in Fig. 8 is bounded by the two analytical estimates of base speed, with the test data being approximately half way between the two estimates. Selecting a base speed of 9000rpm in (14) yields a curve which correlates very well with the test data points. Therefore the base speed of 9000rpm is used in the compensator model, (15).

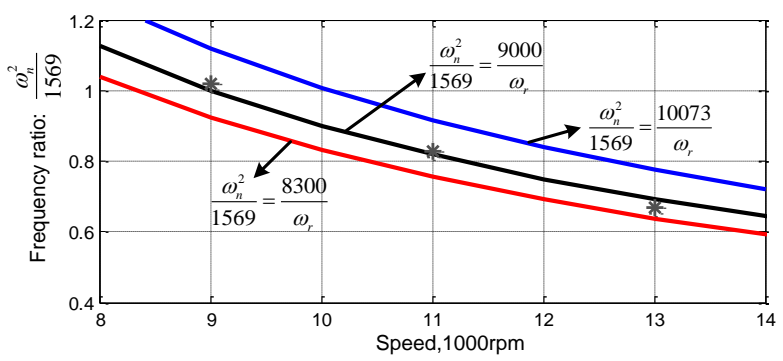

Fig. 8. The relationship between actual speed and frequency ratio

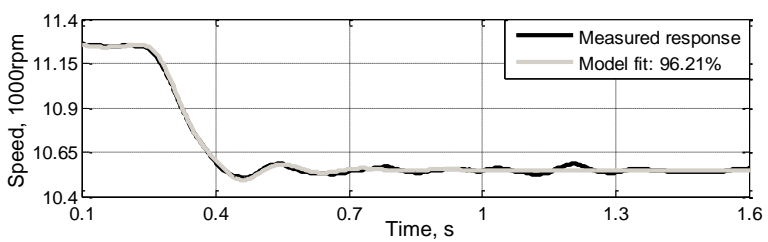

(a) 700rpm step down response from $11250 \mathrm{rpm}$ at $7.29 \mathrm{~kW}$ load

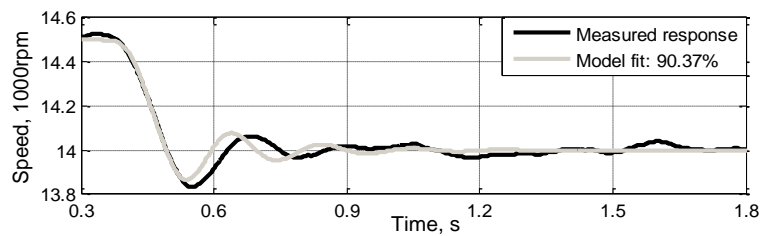

(b) $500 \mathrm{rpm}$ step down response from $14500 \mathrm{rpm}$ at $26.7 \mathrm{~kW}$ load

Fig. 9. Time domain validation test

$$
G_{\text {comp }}= \begin{cases}1+\frac{0.99 s^{2}+s}{0.01 s^{2}+16 s+1569}, & \omega_{r} \leq 9000 \mathrm{rpm} \\ 1+\frac{0.99 s^{2}+s}{0.01 s^{2}+(16 s+1569)} \cdot \frac{\omega_{r}}{9000}, & \omega_{r}>9000 \mathrm{rpm}\end{cases}
$$

When (15) is transformed from the continuous to the discrete domain using the zero-pole matching method with a $1 \mathrm{~ms}$ time delay as used in the RTP the effect on the frequency response up to the maximum bandwidth (120rad/s) of interest is minor and so can be neglected. Note that (15) has been simplified when it is derived from (13) for ease of implementation on the RTP. Frequency analysis has shown that there is no significant difference over the bandwidth of interest.

\section{EXPERIMENTAL COMPENSATOR VALIDATION}

In this section, the identified system model and the developed compensator are validated in both the time and frequency domain for the full speed/power range of the test system.

\section{A. Time Domain Experimental Validation}

The HP spool emulator drive system speed ranges from $8700 \mathrm{rpm}$ to $15000 \mathrm{rpm}$ and the power varies between 0 and 30kW. Fig. 9 shows the identified system model response from (13) overlaid with the measured drive response (after signal pre-filtering). Step down responses at arbitrary load and speed conditions are chosen for cross validation, avoiding conditions 
used for the system model identification. The correlation between the measured and identified model response is excellent in Fig. 9(a). The test in Fig. 9(b) exhibits a slight time delay as the system is approaching the maximum power and speed; however, the correlation between the data sets is still very good. The time delay is due to the saturation of the machine speed controller which is attributed partly to the converter protection designed to avoid magnetic saturation and prevent the motor overheating [24].
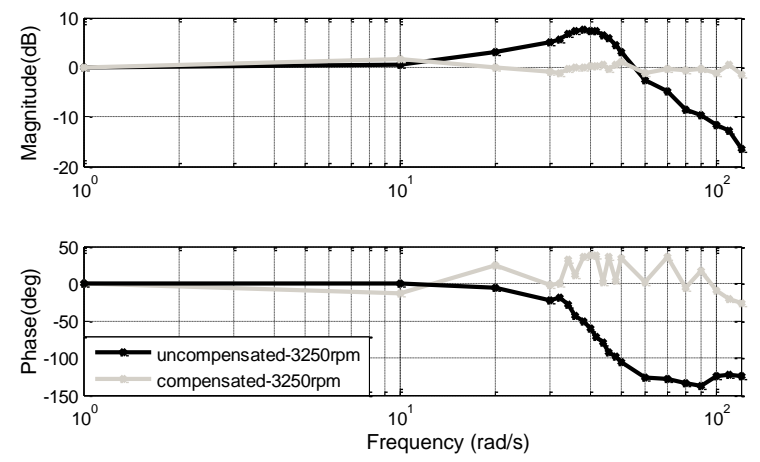

(a) 3250rpm at no load condition
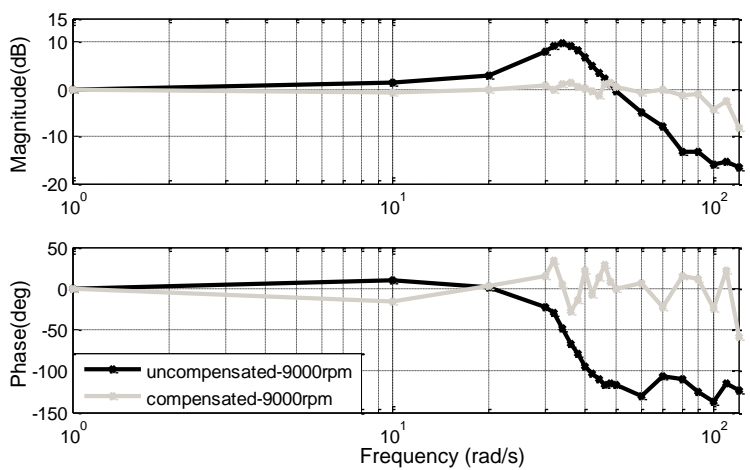

(b) $9000 \mathrm{rpm}$ at $15 \mathrm{~kW}$
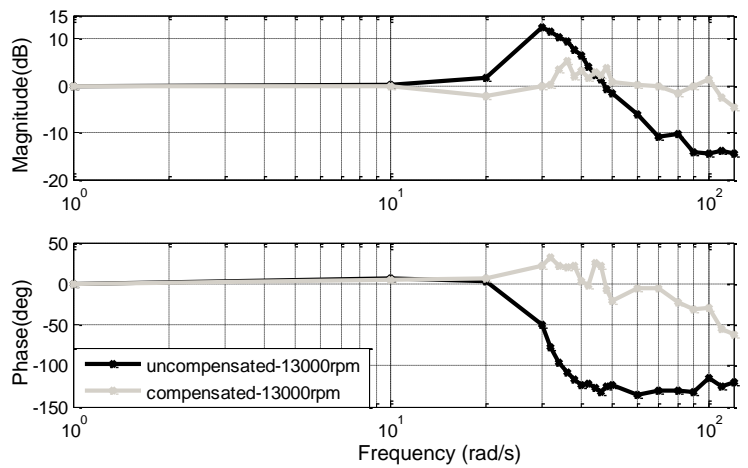

(c) $13000 \mathrm{rpm}$ at $27 \mathrm{~kW}$

Fig. 10. Frequency domain validation results for the developed compensator

\section{B. Frequency Domain Experimental Validation}

For the HP spool emulator drive system, the dynamics are approximately linear over a reasonable frequency range, so the system input-output behavior can be described by the frequency response. Thus, the frequency response validation is often used to assess the identified system model [15] and in this study is applied to validate the developed compensator, (15).
A frequency chirp validation study was performed, sweeping the speed reference to the compensator in Fig. 2, from 10rad/s to $120 \mathrm{rad} / \mathrm{s}$ in $10 \mathrm{rad} / \mathrm{s} \mathrm{steps;} \mathrm{a} 2 \mathrm{rad} / \mathrm{s}$ interval is used between $30 \mathrm{rad} / \mathrm{s}$ and $50 \mathrm{rad} / \mathrm{s}$ to capture the detail around the resonant peak. The chirp amplitude was chosen to be 10rpm (less than $0.1 \%$ of the measurement range), which is the lowest value that can be differentiated from the noise in the signal frequency spectrum.

The experimental results in Fig. 10 illustrate the performance of the system with or without the compensator in the frequency domain. Fig. 10(a) shows the validation results for a machine speed of 3250rpm at no load. The compensator very effectively cancels the resonant peak and so the system bandwidth is doubled from $60 \mathrm{rad} / \mathrm{s}$ to $120 \mathrm{rad} / \mathrm{s}$. The phase difference is also well compensated, for frequencies up to $120 \mathrm{rad} / \mathrm{s}$. The small variation in the measured compensated phase response is due to measurement noise.

Fig. 10(b) and (c) show similar results for two different operating conditions: $9000 \mathrm{rpm}$ at $15 \mathrm{~kW}$ and $13000 \mathrm{rpm}$ at $27 \mathrm{~kW}$. Proper compensation performance is obtained for both cases, significantly extending the system bandwidth. However, the extension of system bandwidth does reduce slightly with operating speed due to the drive's acceleration slew-rate safety feature. This is discussed further in Section IV.C. In Fig. 10(c), the small variation in the measured compensated magnitude response is attributed to the nonlinear saturation effects. However, the variation is still reasonably small, with a peak of $5 \mathrm{~dB}$ in comparison with the uncompensated case peak of almost $14 \mathrm{~dB}$.

\section{Effect of Slew Rate Limit}

A conservative slew rate limit of $393.7 \mathrm{rad} / \mathrm{s}^{2}$ has been set in the drive system to protect the system and personnel. For the compensator to be effective, the compensator output must fall within the drive's inherent slew rate limit, $K_{S}$, so:

$$
A \cdot B \cdot \omega \leq K_{S}
$$

where $A$ is the variable frequency signal amplitude, $\omega$ is the signal frequency and $B$ is the gain of the compensator. Therefore, for a specific emulated mechanical dynamics with a bandwidth $\omega$ and amplitude $A$, the compensator's gain $B$ is limited to $K_{S} /(A \cdot \omega)$. Beyond this gain limit, the effectiveness of the compensator will be impaired. In addition, as the machine speed increases, the corresponding effective frequency range of the compensator, which is restricted by the compensator gain limit, decreases. In this emulation system, $K_{S}$ is $393.7 \mathrm{rad} / \mathrm{s}^{2}$, the frequency range of interest has a maximum value of $120 \mathrm{rad} / \mathrm{s}$ and $A$ is set at $10 \mathrm{rpm}$, so $B$ is 3.13 , which corresponds to $9.9 \mathrm{~dB}$ in this chirp test.

In Fig. 10, as the test speed increases, the compensator's effective frequency range decreases accordingly. For the 3250rpm case in Fig. 10(a), the compensated system response has a magnitude and phase angle of approximately zero within the whole test region. Zero magnitude and phase angle can be maintained until the frequency reaches $110 \mathrm{rad} / \mathrm{s}$ for the 9000rpm case in Fig. 10(b) while it is $100 \mathrm{rad} / \mathrm{s}$ for the 13000rpm case in Fig. 10(c). These results confirm the validity of the bandwidth limit analysis for the developed compensator. 
The current loop dynamics may also impose a limit on the emulated mechanical dynamics, however, as the current loop bandwidth is $1100 \mathrm{~Hz}$ the drive slew rate limit dominates the overall bandwidth of the emulated mechanical system. The slew rate limit could be increased by using a converter with a higher current rating, up to the thermal and saturation limit of the induction motor, but this may increase the system cost. Increasing the machine rating would increase both torque and inertia, giving at most marginal gains on slew rate and hence compensator performance.

\section{Aero Gas Engine Dynamics Emulation}

To illustrate the effectiveness of the proposed compensator, it is employed to enhance the emulation of an aero gas engine by accurately reproducing the dynamic response of the engine and fuel controller.

The gas engine model is a two-spool aero gas engine. The environment data (altitude and Mach number) was set to the default values for this investigation. Throttle (pilot lever angle (PLA)) demands and electrical power extraction commands are normally used to assess the aero engine's performance, where a step change in commanded PLA is the most demanding scenario [25]. Therefore, the experimental and simulation tests of the PLA step change and generator load steps are used to validate the proposed emulation technique for this study. The simulation study is performed using a transient simulation model (using the parameters in the Appendix) of the full HP spool emulation facility in Fig. 1 and a gas engine model. As the compensator (15) is developed for the experimental test rig, which is different from the theoretical system model, a simulation model based compensator is developed separately using the method described in Section III.

In Fig. 11, a PLA step change between $58^{\circ}$ and $60^{\circ}$ (the maximum angle) is applied to the engine model at no load condition. The uncompensated emulation performance is shown in Fig. 11(a) while the compensated response is in Fig. 11(b). The corresponding torque response is shown in Fig. 11(c). The measured and simulated responses show close correspondence. When the PLA step change is applied, the engine dynamics have an approximately 30rad/s oscillation during the dynamic change. Fig. 10 indicates that such an oscillation is around the resonant peak of the uncompensated system. Due to the high gain at the resonant frequency, the speed input is amplified significantly and hence the uncompensated speed response has a higher overshoot and a longer settling time in comparison with the engine model reference. However, when the compensator is active the system resonant peak is accurately compensated, therefore, the emulator speed response is significantly improved, enabling excellent dynamic speed tracking.

Fig. 12 and 13 describe the compensator performances for replicating the engine dynamics to a $38^{\circ}$ to $40^{\circ}$ and $58^{\circ}$ to $59^{\circ}$ PLA step change at $27 \mathrm{~kW}$. When the generator (SRSG) is fully loaded, the uncompensated system responses exhibit a more oscillatory under-damped response than that seen in the no load PLA test, especially for the high PLA test. When the machine drive is operating at nearly the full power condition, the motor needs to produce a much higher torque due to the high load and much amplified speed dynamics, which is beyond the torque limit, in order to track the engine speed during the PLA step change.

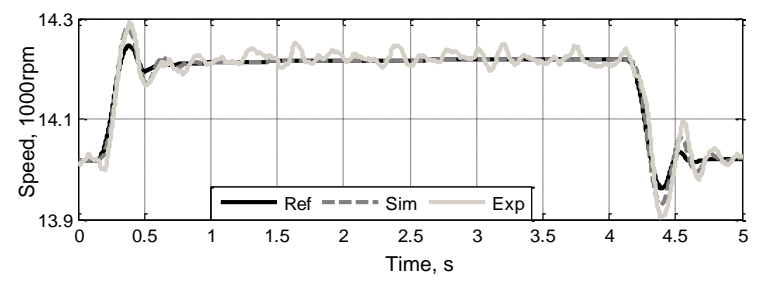

(a) Compensator disabled

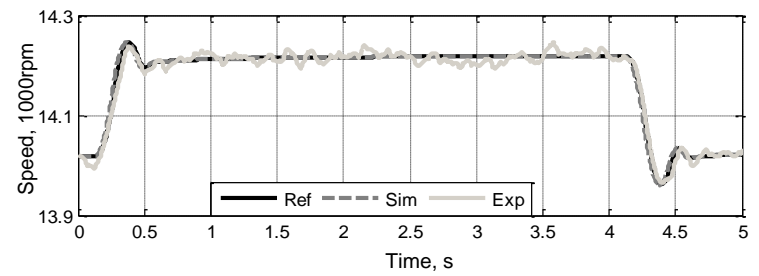

(b) Compensator enabled

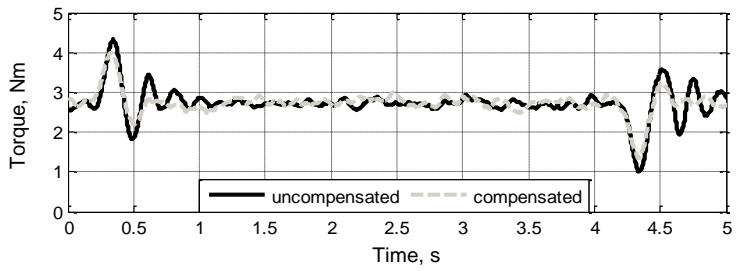

(c) Experimental system torque responses

Fig. 11. Compensator performance for engine dynamics to 58\%-60\% PLA step change at no load.

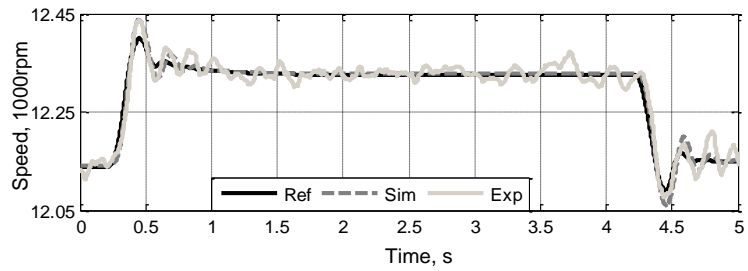

(a) Compensator disabled

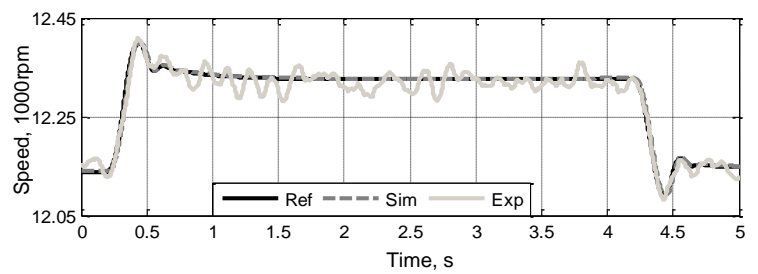

(b) Compensator enabled

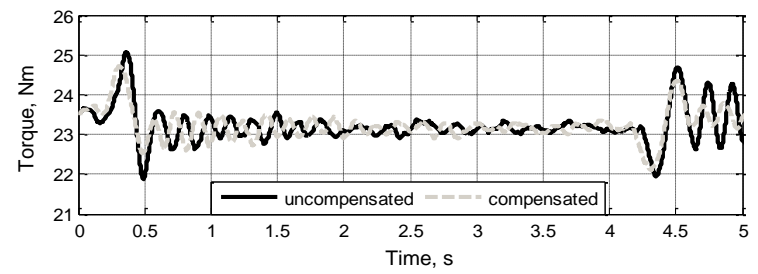

(c) Experimental system torque responses

Fig. 12. Compensator performance for engine dynamics to $38 \%-40 \%$ PLA step change at full power. 


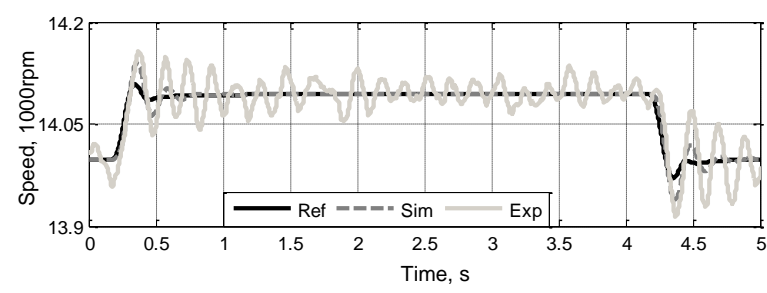

(a) Compensator disabled

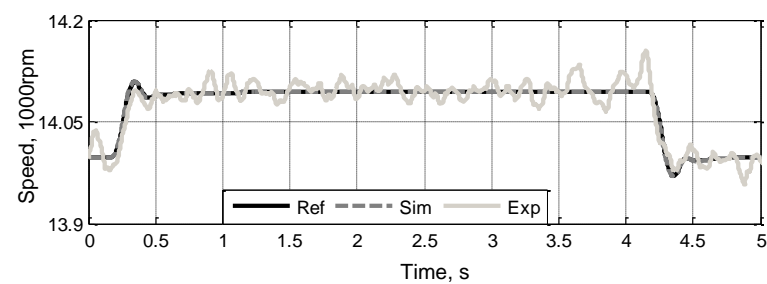

(b) Compensator enabled

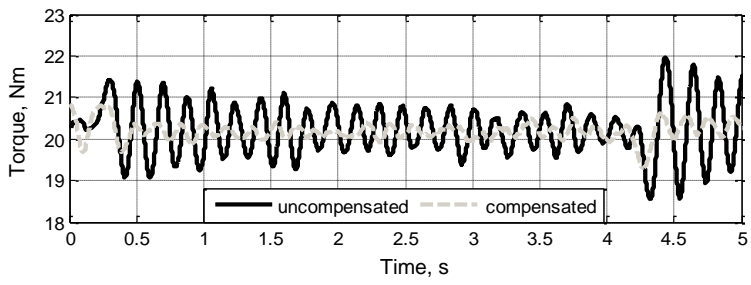

(c) Experimental system torque responses

Fig. 13. Compensator performance for engine dynamics to 58\%-59\% PLA step change at full power

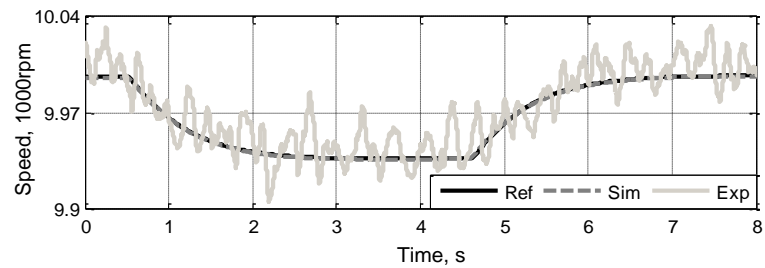

(a) Compensator disabled

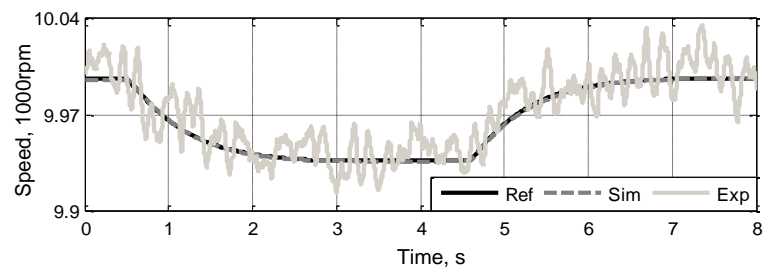

(b) Compensator enabled

Fig. 14. Compensator performance for engine dynamics to electrical power transients

The over-limit torque requirement would make the drive current response saturate, Fig. 12(c) and Fig. 13(c), which is associated with the observed speed oscillations, Fig. 12(a) and Fig. 13(a). A similar saturation phenomenon is also identified in [9] and attributed to the motor torque-producing limitation. However, with the compensator in place, the drive system dynamics are well compensated to make the drive torque requirement become within the limit. Therefore the actual machine speed is tracking the engine reference, Fig. 12(b) and Fig. 13(b), and the oscillations are well damped, indicating that the compensator is effective in eliminating such a saturation effect, which extends the system emulation capability. The results, along with Fig. 11, verify the effectiveness of the proposed compensator in the high speed and high power applications emulation and confirm the validity of the frequency analysis in Section IV.

Fig. 14 shows the speed tracking performance of the HP spool emulator motor drive during a $15 \mathrm{~kW}$ electrical load step; the power increases at $\mathrm{t}=0.5 \mathrm{~s}$ and decreases at $\mathrm{t}=4.5 \mathrm{~s}$. The drive speed controller is designed to give accurate tracking of the engine speed responses during the power transients. In Fig. 14, the settling time for the engine to the load step is $3 \mathrm{~s}$ which is well within the effective bandwidth of the uncompensated system (approximately 10rad/s). Therefore, the emulator speed correlates well with the reference speed from the engine model with or without the compensator for both power step up and step down scenarios.

\section{CONCLUSIONS}

A simple, easily-implemented technique for mechanical system emulation is proposed. This method is based on the development of a compensator, which can be fully parameterised using simple system transient step tests. The technique is particularly useful for fast dynamics emulation at high speeds, and high load where full drive system parameters are not available.

The compensator dependency on the system speed and load operating conditions has been fully analysed. Experimental validation results have shown excellent accuracy of the identified system model over the full operating range of the test rig. The electrical drive based emulation of an aero gas engine has also yielded good equivalence for the whole engine operating range and also controller nonlinear saturation region, for which it would be very difficult to achieve such an agreement using the analytical derived compensator method.

The proposed emulation scheme is generic and not fixed to a specific mechanical system. This study has been focused on the mechanical emulation of a high inertia power source, in this case an aero gas engine. However, for the gas engines having very high power density with much smaller inertias such as the aeroderivative gas turbines, the approach can also allow a good emulation without redesigning the emulation technique.

The compensator performance is enabled by the emulator drive rating, which is associated with the drive inherent slew rate. The investigation of the impact of the drive rating on the compensator performance, as well as the emulation of high order systems such as mechanical drivetrain and backlash effects, will be the subject of future publications.

\section{ACKNOWLEDGMENT}

The authors are grateful to The University of Manchester Alumni Fund and the School of Electrical and Electronic Engineering for the studentship support. The authors would like to thank Rolls-Royce plc for promoting and funding the Intelligent Electrical Power Network Evaluation Facility (IEPNEF) which is housed at The University of Manchester in the School of Electrical and Electronic Engineering. 
APPENDIX

TABLE I

HP SPOOL EMULATOR DRIVE SETTINGS

\begin{tabular}{l|l}
\hline Parameter & Value \\
\hline Speed controller proportional gain, $K_{P}$ & 4.5 \\
Speed controller integral gain, $K_{I}$ & 270 \\
Current controller proportional gain, $K_{P I}$ & 36 \\
Current controller integral gain, $K_{I I}$ & 116 \\
Machine torque constant, $K_{T}$ & $0.85 \mathrm{Nm} / \mathrm{A}$ \\
\hline
\end{tabular}

TABLE II

PARAMETERS FOR THE INDUCTION MOTOR (REFERRED TO STATOR SIDE)

\begin{tabular}{l|l}
\hline Parameter & Value \\
\hline Stator winding resistance, $R_{s}$ & $10.5 \mathrm{~m} \Omega$ \\
Rotor winding resistance, $R_{\mathrm{r}}$ & $8.6 \mathrm{~m} \Omega$ \\
Stator leakage reactance, $X_{l s}$ & $78 \mathrm{~m} \Omega$ \\
Rotor leakage reactance, $X_{l r}$ & $47.5 \mathrm{~m} \Omega$ \\
Magnetizing reactance, $X_{m}$ & $4.85 \Omega$ \\
Rated voltage, $V_{\text {rated }}$ & $400 \mathrm{~V}$ \\
Pole pair, $p p$ & 1 \\
Rated speed, $\omega_{\text {rated }}$ & $6745 \mathrm{rpm}$ \\
Rated frequency, $f_{\text {rated }}$ & $113.5 \mathrm{~Hz}$ \\
Test rig inertia, $J( \pm 10 \%$ accuracy $)$ & $0.11 \mathrm{~kg} \cdot \mathrm{m}^{2}$ \\
Damping coefficient, $B$ & $0.0018 \mathrm{~N} . \mathrm{m} . \mathrm{s}$ \\
\hline
\end{tabular}

\section{REFERENCES}

[1] C. Gan, R. Todd, and J. Apsley, "Time-delay effects in a HIL aircraft power system emulator," in Proc. of IEEE Energy Conversion Congress and Exposition (ECCE), pp. 20-26, 2013.

[2] B. A. Correa, Y. Zhang, R. Fang, and R. A. Dougal, "Driving a synchronous motor so that it emulates a twin-shaft gas turbine engine," in Proc. of the 6th IET Power Electronics, Machines and Drives (PEMD) conference, Mar.26-29, 2012.

[3] Z. Hakan Akpolat, G. M. Asher, and J. C. Clare, "Dynamic emulation of mechanical loads using a vector-controlled induction motor-generator set," IEEE Trans. Ind. Electron, vol. 46, pp. 370-379, 1999.

[4] H. M. Kojabadi, L. Chang, and T. Boutot, "Development of a novel wind turbine simulator for wind energy conversion systems using an inverter-controlled induction motor," IEEE Trans. Energy Conv, vol. 19, pp. 547-552, 2004.

[5] H. Li, M. Steurer, K. L. Shi, S. Woodruff, and D. Zhang, "Development of a unified design, test, and research platform for wind energy systems based on hardware-in-the-loop real-time simulation," IEEE Trans. Ind. Electron, vol. 53, pp. 1144-1151, 2006.

[6] M. Rodic, K. Jezernik, and M. Trlep, "Dynamic emulation of mechanical loads: an advanced approach," IEE Proceedings-Electric Power Applications, vol. 153, pp. 159-166, 2006.

[7] R. Todd and A. J. Forsyth, "HIL emulation of all-electric UAV power systems," in Proc. of IEEE Energy Conversion Congress and Exposition (ECCE), pp. 411-416, 2009.

[8] E. J. Thomas and P. W. Sauer, "Engine-simulation control of an induction motor," in Proc. of Electrical Insulation Conference and Electrical Manufacturing \& Coil Winding Conference, pp. 589-594, 1999.

[9] B. A. Correa, Y. Zhang, R. A. Dougal, T. Chiocchio, and K. Schoder, "Mechanical Power-Hardware-in-the-Loop: Emulation of an Aeroderivative Twin-Shaft Turbine Engine," in Proc. of IEEE Electric Ship Technologies Symposium (ESTS), pp. 464-468, 2013.

[10] L. A. C. Lopes, J. Lhuilier, and M. F. Khokhar, "A wind turbine emulator that represents the dynamics of the wind turbine rotor and drive train," in Proc. of IEEE Power Electronics Specialists Conference (PESC), pp. 2092-2097, 2005

[11] B. Rabelo, W. Hofmann, and M. Gluck, "Emulation of the static and dynamic behaviour of a wind-turbine with a DC-machine drive," in Proc. of IEEE Power Electronics Specialists Conference (PESC), pp. 2107-2112, 2004.

[12] E. R. Collins Jr and Y. Huang, "A programmable dynamometer for testing rotating machinery using a three-phase induction machine," IEEE Trans. Energy Conv, vol. 9, pp. 521-527, 1994.

[13] Z. H. Akpolat, G. M. Asher, and J. C. Clare, "Experimental dynamometer emulation of nonlinear mechanical loads," IEEE Trans. Ind. Appl, vol. 35, pp. 1367-1373, 1999.
[14] J. Arellano-Padilla, G. M. Asher, and M. Sumner, "Control of an AC dynamometer for dynamic emulation of mechanical loads with stiff and flexible shafts," IEEE Trans. Ind. Electron, vol. 53, pp. 1250-1260, 2006.

[15] L. Ljung, "System Identification: Theory for the user, 2nd ed", Prentice Hall, 1999.

[16] L. Amedo, R. Burgos, F. Wang, and D. Boroyevich, "Black-box terminal characterization modeling of dc-to-dc converters," in Proc. of IEEE Applied Power Electronics Conference (APEC), pp. 457-463, 2007.

[17] L. Ljung, "System Identification Toolbox 7", User's Guide, The Mathworks Inc. Natick, MA, 2007.

[18] V. Valdivia, A. Barrado, A. Lazaro, M. Sanz, D. Lopez del Moral, and C. Raga, "Black-Box Behavioral Modeling and Identification of DC-DC Converters with Input Current Control for Fuel Cell Power Conditioning," IEEE Trans. Ind. Electron, vol. 61, pp. 1891-1903, 2014.

[19] S. R. Krishnan and C. S. Seelamantula, "On the Selection of Optimum Savitzky-Golay Filters," IEEE Trans. Signal Processing, vol. 61, pp. 380-391, 2013

[20] V. Valdivia, R. Todd, F. Bryan, A. Barrado, A. Lazaro, and A. Forsyth, "Behavioural Modelling of a Switched Reluctance Generator for Aircraft Power Systems," IEEE Trans. Ind. Electron, vol. 61, pp. 2690-2699, 2014

[21] L. Arnedo, D. Boroyevich, R. Burgos, and F. Wang, "Polytopic Black-Box modeling of DC-DC converters," in Proc. of IEEE Power Electronics Specialists Conference (PESC), pp. 1015-1021, 2008.

[22] S.-H. Kim and S.-K. Sul, "Voltage control strategy for maximum torque operation of an induction machine in the field weakening region," IEEE Trans. Ind. Electron, vol. 44, pp. 512-518, 1997.

[23] M. Mengoni, L. Zarri, A. Tani, G. Serra, and D. Casadei, "A Comparison of four robust control schemes for field-weakening operation of induction motors," IEEE Trans. Power Electron, vol. 27, pp. 307-320, 2012.

[24] H.-B. Shin, "New antiwindup PI controller for variable-speed motor drives," IEEE Trans. Ind. Electron, vol. 45, pp. 445-450, 1998

[25] C. Miller, J. Zumberge, and M. Wolff, "Hardware-in-the-Loop Electric Drive Stand Issues for Jet Engine Simulation," in SAE International Power Systems Conference, 2010.

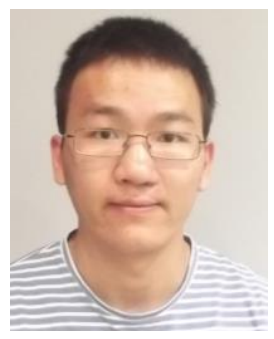

Chengwei Gan received the B.Eng. degree from North China Electric Power University (NCEPU), China, and the first class B.Eng. (Hons.) degree from The University of Manchester, Manchester, U.K., in 2010, where he is currently working toward the Ph.D. degree.

His research interests include time-delay compensation techniques for use in industrial control platforms, electrical drive based mechanical system emulation and more electric aircrafts.

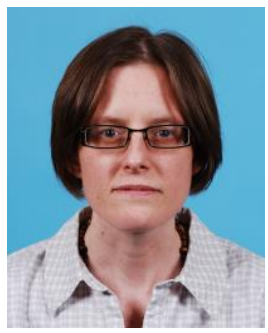

Rebecca Todd (M'08-SM'12) received the M.Eng. degree from the University of Manchester Institute of Science and Technology, Manchester, U.K., in 2001 and the Eng.D. degree from the University of Manchester, Manchester, U.K., in 2006.

She was a Research Associate in the Rolls-Royce University Technology Centre from 2006 to 2010, and since 2010 has been a Lecturer at The University of Manchester. Her research interests include advanced control methods, supercapacitor-based energy storage devices and energy management for on-board electrical systems comprising multiple, engine embedded generators and power electronic motor drive loads.

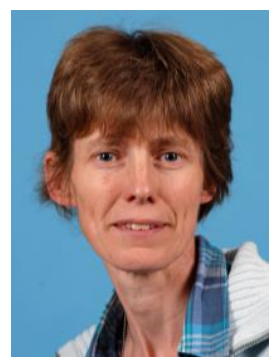

Judith M. Apsley received her BA degree from the University of Cambridge, UK in 1986 and her Ph.D. from the University of Surrey, UK in 1996. She worked at Westland Helicopters (1982-1987), ERA Technology (1987-1995) and a small software company (1996-2001), before joining the University of Manchester as a Research Associate, and was appointed as a lecturer in 2008. Her main research interests are the modelling and control of electrical drive and generator systems for aircraft and marine applications 